\title{
An Enhanced Feature Extraction Method and Classification Method of EEG Signals using Artificial Intelligence
}

\author{
Shilpa Bharti \\ Student, M.Tech, Electronics and Communication, \\ Rayat\&Bahra institute of Engineering and Bio- \\ technology, Mohali, India
}

\author{
Sukhman Preet Singh \\ Assistant Professor, Department of Electronics and \\ Communication Rayat\&Bahra Institute of \\ Engineering \& Bio-Technology, Mohali, India
}

\begin{abstract}
Emotion Recognition from EEG signs permits the immediate appraisal of the "internal" condition of a client, which is viewed as an essential figure human-machine-connection. Numerous systems for feature extraction have been mulled over. Their suitability for emotion recognition, be that as it may, has been tried utilizing a little measure of particular capabilities and on distinctive, typically little information sets. In the proposed work NN based Classification will be done on EEG Signal dataset that has been collected from FORTIS HOSPITAL AND BCI Competition. First feature extraction was applied to the raw data. Then the resulted feature vectors were used to train the classifiers. At last the classifiers were tested with the data not seen during the training to evaluate their classification accuracy. The results indicate that the $\mathrm{NN}$ classifier produces best classification accuracy than genetic algorithm.
\end{abstract}

\section{Keywords}

Emotion Recognition, EEG Signal, Feature Extraction, Classification, Neural network, BCI, FAR, FRR

\section{INTRODUCTION}

Recently, there has been a lot of interest in the area of Brain Computer Interface (BCI) research as it has the potential to provide communication and control capabilities to people with severe motor disabilities [1]. This is a multi-disciplinary research including specialists from neurobiology, brain research, building, arithmetic, and software engineering [2,3]. Any functional execution of BCI configuration requires an effective signal processing plan that incorporates signal preprocessing, feature-extraction and characterization [4].

Endeavors in human-machine-interaction (HMI) go for discovering approaches to better and all the more fittingly interface with PCs/people [5]. To make HMI more normal, learning about the enthusiastic condition of a client is viewed as an essential element. Emotions are essential for right understanding of activities and in addition correspondence. Enthusiasm for emotion recognition from distinctive modalities (e.g. face, stance, movement, and voice) has ascended in the previous decade and as of late picked up consideration in the field of brain computer-interfaces (BCIs), which has authored the term full of feeling $\mathrm{BC}[6,7,8]$.

In this paper, we stress on the classification and feature extraction part so it will be useful in emotion recognition [9]. Preferably, a great classifier for BCI ought to deliver high classification accuracy with minimal classification complexity and feature extraction procedure must concentrate includes accurately. In a matter of seconds, various direct and nonlinear classifiers have been suggested for this application. They incorporate measurable techniques, for example, Linear Discriminant Analysis (LDA), Hidden Markov Classifier and z-scale base Discriminant Analysis (ZDA) [10]. The fundamental downside of these techniques is that they don't function admirably for nonlinear order issues. As of late backpropagation neural systems (BPNNs) and Support vector machines (SVMs) have been indicated to enhance the classification accuracy and linear methods, for example, Linear discriminant analysis $[11,12]$.

So, in this proposed work NN based Classification will be done on EEG Signal dataset that has been collected from forties hospital and from V BCI Competition III. First feature extraction was applied to the raw data [13]. Then the resulted feature vectors were used to train the classifiers. At last the classifiers were tested with the data not seen during the training to evaluate their classification accuracy. The results indicate that the NN classifier produces similar classification accuracy but requires more precisely feature extraction, so for feature extraction genetic algorithm used. [14].

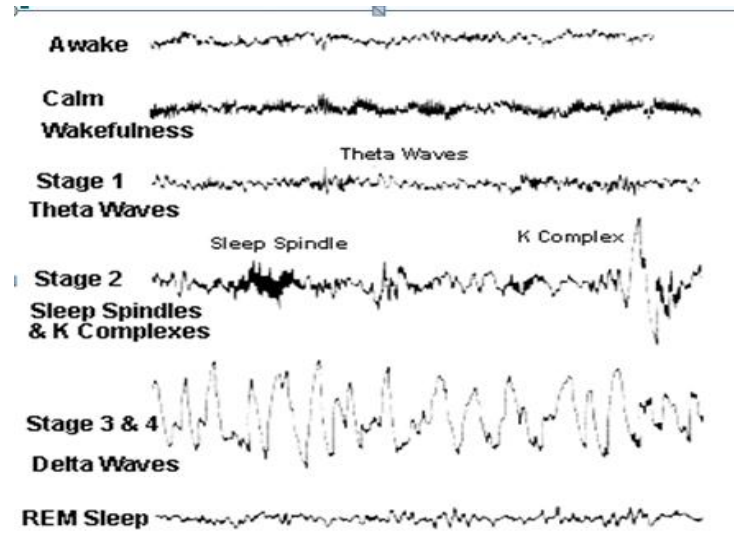

Figure 1 Different waves in EEG

Alpha wave frequency from $8-13 \mathrm{hz}$ and amplitude is $20-60$ $\mu \mathrm{V}$. Easily produced when quietly sitting in relaxed position with eyes closed (few people have trouble producing alpha waves). Beta waves frequency is from 14-30 hz and amplitude is $2-20 \mu \mathrm{V}$. The most common form of brain waves are present during mental thought and activity. Theta wave frequency lies from $4-7 \mathrm{hz}$ and amplitude is from $20-100 \mu \mathrm{V}$. Believed to be more common in children than adults. Walter Study (1952) found these waves to be related to displeasure, pleasure, and drowsiness. Delta waves lies between 5 to $3.5 \mathrm{hz}$ and amplitude lies in $20-200 \mu \mathrm{V}$. Found during periods of 
deep sleep in most people. Very irregular and slow waves pattern.

The paper is organized as follows. Section 2 gives a description of the EEG Signal classification problem. Section 3 presents the details of the proposed algorithm Section 4 gives the Performance evaluation using parameters. Conclusion and future scope from this study are summarized in Section 6.

\section{EEG SIGNAL CLASSIFICATION}

Below are the steps to compute the classification problem using AFNN and genetic algorithm .

\subsection{Measuring EEG}

EEG database has been collected from forties hospital and from V BCI Competition III.

For EEG dataset the electrode should be connected to head. The electrodes are connected to head by a EEG gel. In order to avoid artifacts, the participants should be in a relaxed mode. At last signals are recorded in raw form.

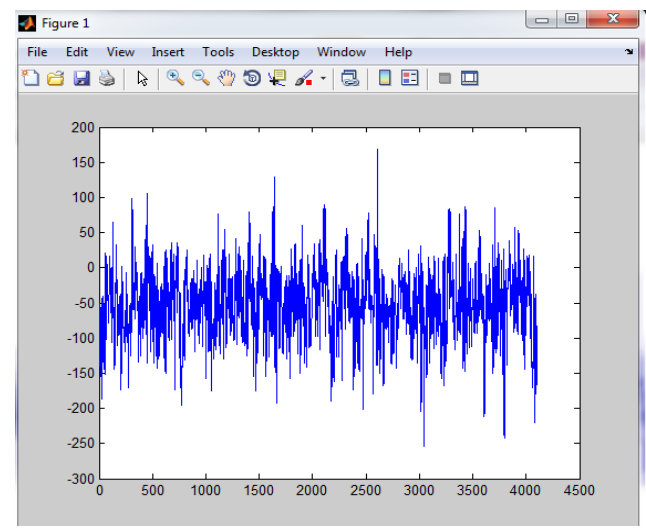

Figure 2 Normal signal of EEG

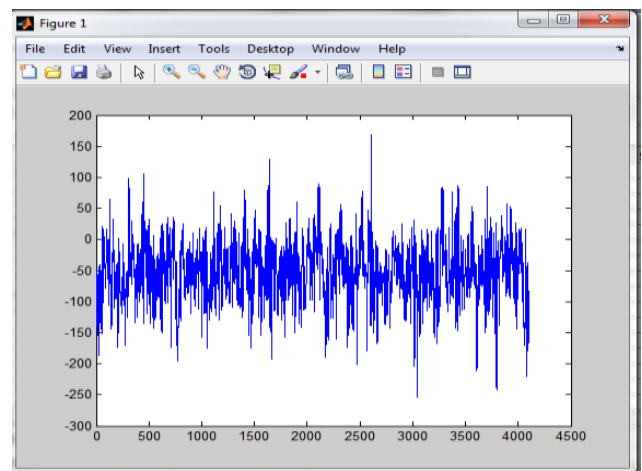

Figure 3 Abnormal signal of EEG

\subsection{Feature extraction}

Feature extraction of EEG signals mainly done using features like Time domain, Frequency Domain and Time-Frequency Domain. EEG signals are the rich source of information about brain activity. The information in EEG signals also includes information about emotions [15]. This has to extract the valuable information from the large amount of data. For this task, the system will first reduce the amount of data available. This process is known as feature extraction and extracts specified measures that are useful for the task. These features should contain enough information about the emotion. After reducing the size of the data, the emotion has to be detected from the features. The following processes are involved in successful emotion detection.

\subsection{Optimization using genetic algorithm}

Genetic algorithms are motivated by Darwin's hypothesis about development. Answer for an issue explained by genetic algorithms is advanced. Algorithm s begun with a situated of arrangements (spoke to by chromosomes) called population. Arrangements from one populace are taken and used to frame another population.

1. [Start] Produce arbitrary population of $n$ chromosomes (suitable answers for the issues).

2. [Fitness] Assess the fitness $f(x)$ of every chromosome $x$ in the population.

3. [New population] Make another populace by rehashing after ventures until the new populace is finished.

a) [Selection] Select two guardian chromosomes from a populace as indicated by their wellness (the better wellness, the greater opportunity to be chosen).

b) [Crossover $]$ With hybrid likelihood traverse the folks to shape posterity (kids). On the off chance that no hybrid was performed, posterity is an accurate duplicate of folks.

c) [Mutation] With a transformation likelihood change new posterity at every locus (position in chromosome).

d) [Accepting] Put new posterity in another populace.

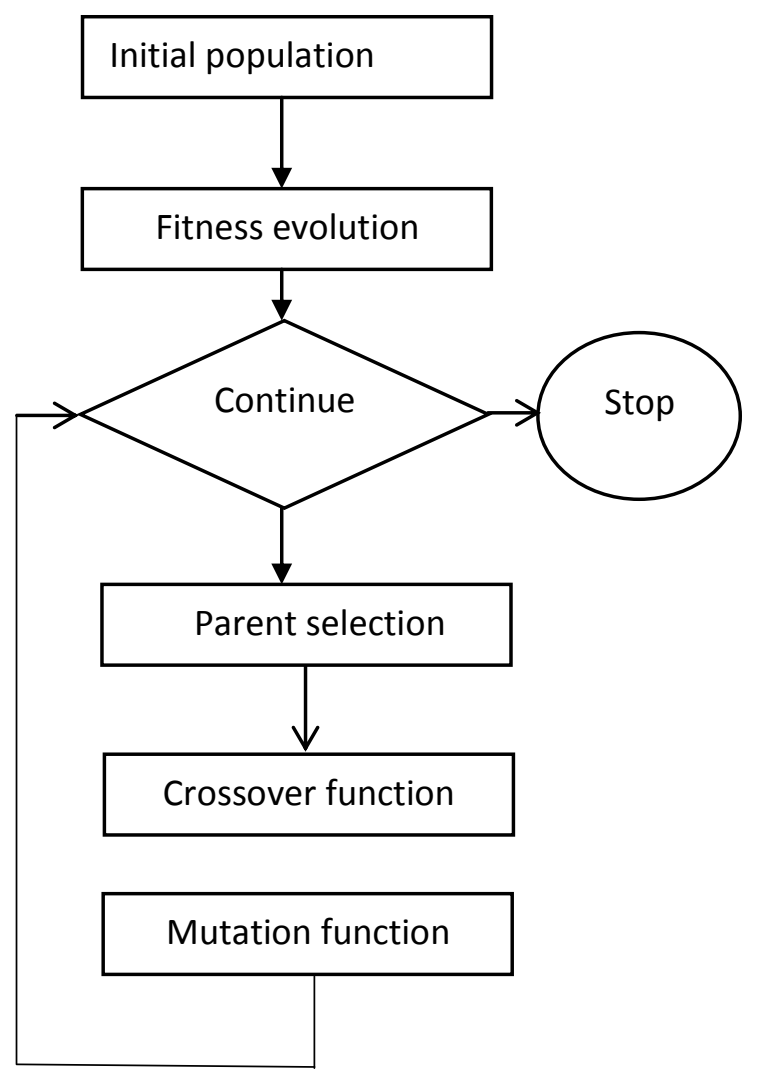

Figure 4 Flowchart of genetic algorithm 
4. [Replace] Utilization new produced population for a further run of algorithm.

5. [Test] If the end condition is fulfilled, stop, and return the best arrangement in current population.

[Loop] Go to step 2.

\subsection{Classification}

The classification of feature extracted method has been done using AFNN classifier. The AFNN works in the form of weights and the output values can be changes according to the weights inputted in the system.

Artificial neural network can be described as:

$\mathrm{Y}(\mathrm{k} 1)=\mathrm{F}\left(\sum_{i=0}^{m 1}(\mathrm{wi}) \mathrm{k} 1 \cdot \mathrm{xi} k 1+b\right)$

Where: Xik1 is input value in discrete time.

Wi $\mathrm{k} 1$ is weight value in discrete time

$\mathrm{B}$ is bias,

$\mathrm{F}$ is a transfer function,

$\mathrm{Y}(\mathrm{k})$ is output value in discrete time

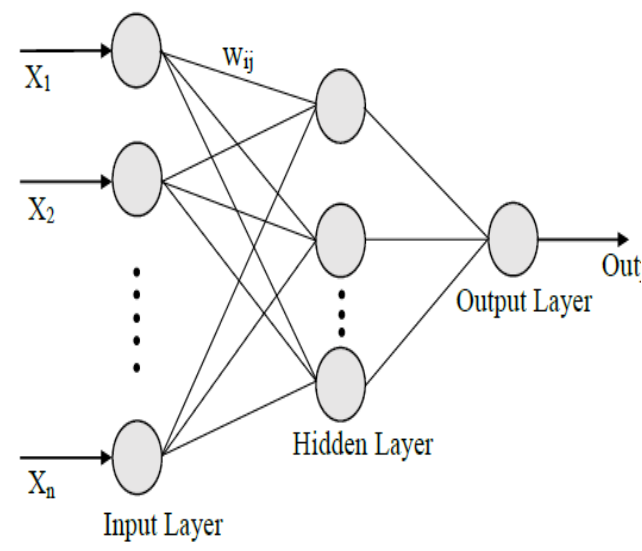

Figure 5 Neural Networks architecture

\section{PROPOSED WORKING}

Flowchart describes the steps that have been followed to classify the EEG signals into normal or abnormal signal of EEG.

In this genetic algorithm is used for classification and also for the comparison of accuracy with neural network.

Table no.1 Parameters of GA

\begin{tabular}{|l|l|}
\hline Population size & 50 \\
\hline Mutation & 0.05 \\
\hline Crossover & 0.8 \\
\hline Feature selected & 1 to 100 \\
\hline
\end{tabular}

EEG signals are the rich source of information about brain activity. However, since brain activity produces very weak signals and the EEG signals contain a lot of background noise therefore before using the signals for emotion detection, they have to be preprocessed, in order to remove unwanted noise.
The information in EEG signals also includes information about emotions. Below are the steps to compute the classification problem using AFNN and genetic algorithm.

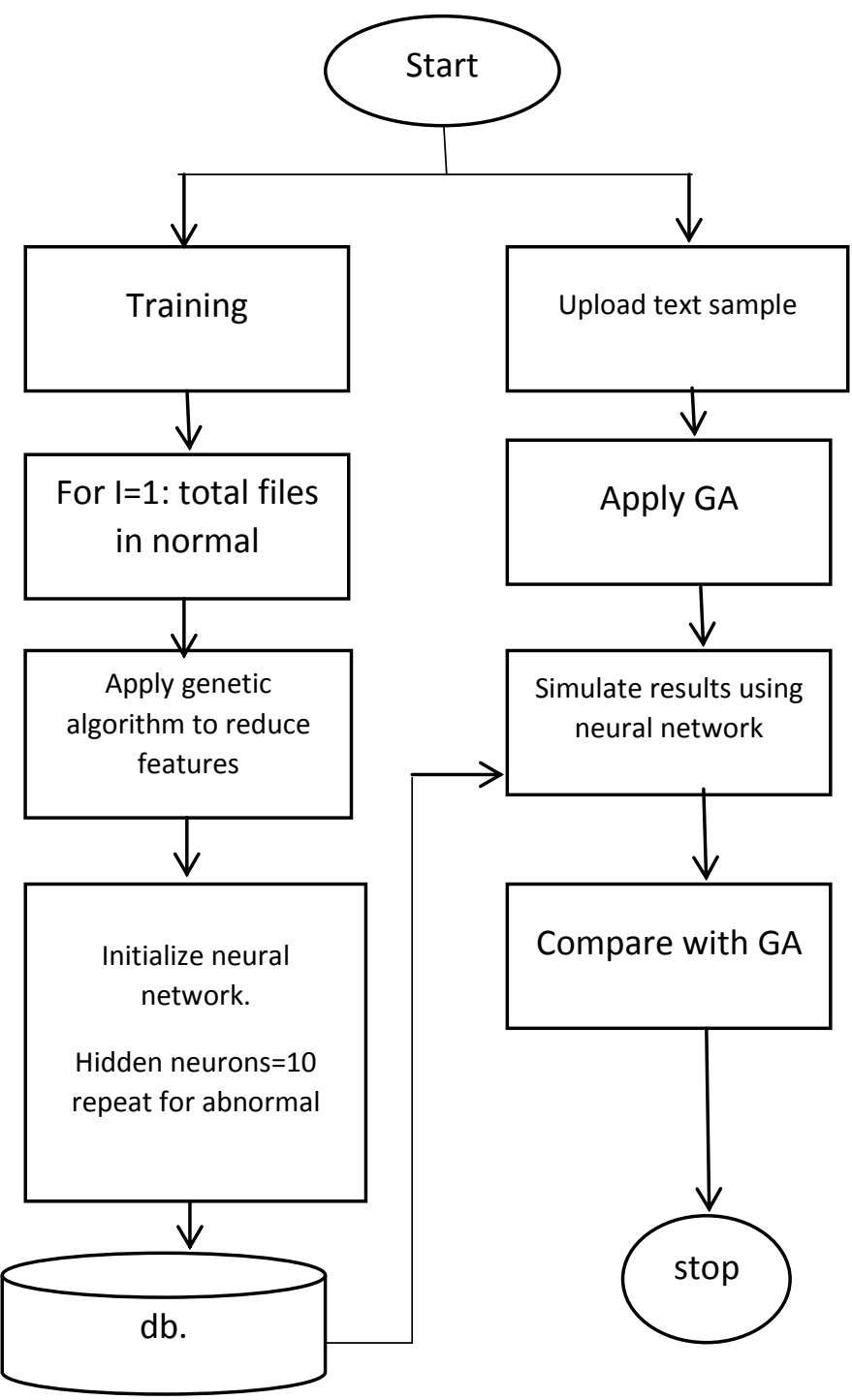

Figure 6 Proposed work Flowchart

\section{RESULTS AND IMPLEMENTATION}

The whole implementation has been taken place in MATLAB environment. Below figures shows the working of the proposed algorithm.

The testing of uploaded text file in which no. $f$ epochs taken are 50, gradient value is $1.22, \mathrm{Mu}$ value is, .001 and 6 validation checks has been used to test the uploaded file.

Performance plots for EEG signals: - performance plot shows mean sq. Error dynamics for all the data sets in logarithmic scale.

Training MSE is always decreasing. Validation fails are iteration. MATLAB automatically stops training after 6 fails in row. Regression gives the idea of how close the output from your model to the actual target values. Mu is the training gain or control parameter of the algorithm. 


\subsection{Feature extraction of normal signal and abnormal signal of EEG in time and frequency domain}

Feature of EEG signals can be extracted in time domain as well as frequency domain. In this work features are extracted in both domains. In time domain feature extraction a sine wave forms. This sine wave represents with one cycle per second..

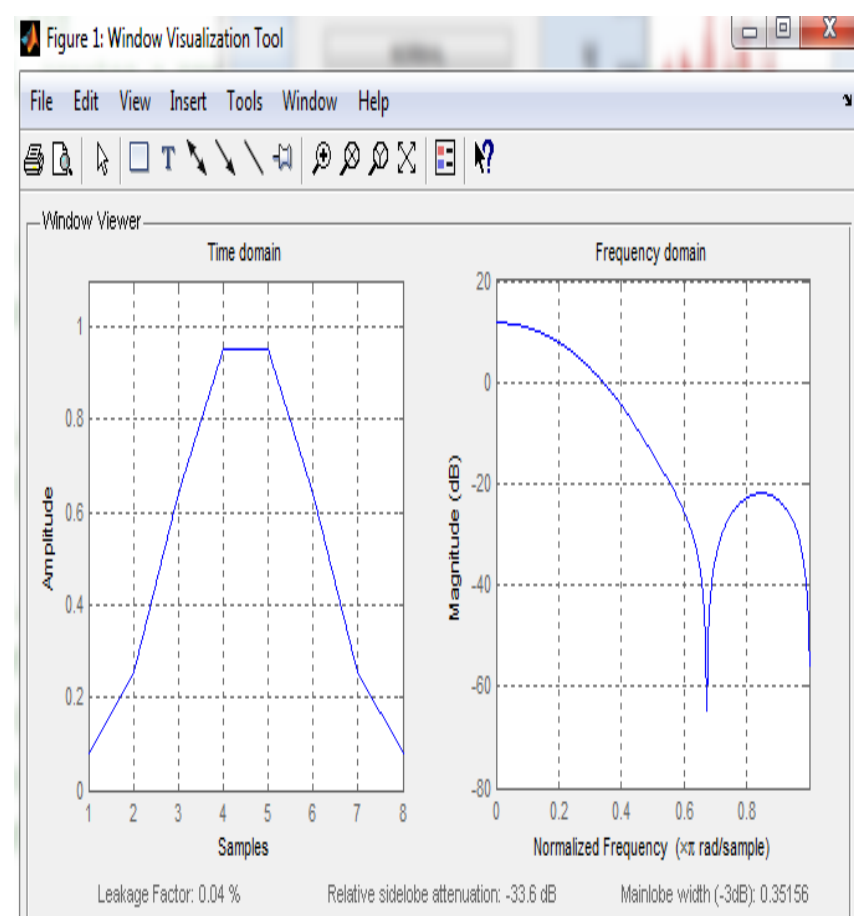

Figure no.7 feature extraction of normal signal

In frequency domain peak of the signal represent frequency component of sine wave. The tail of signal represents the artifacts on signal.

Frequency domain method may not provide high quality performance for some EEG signal.

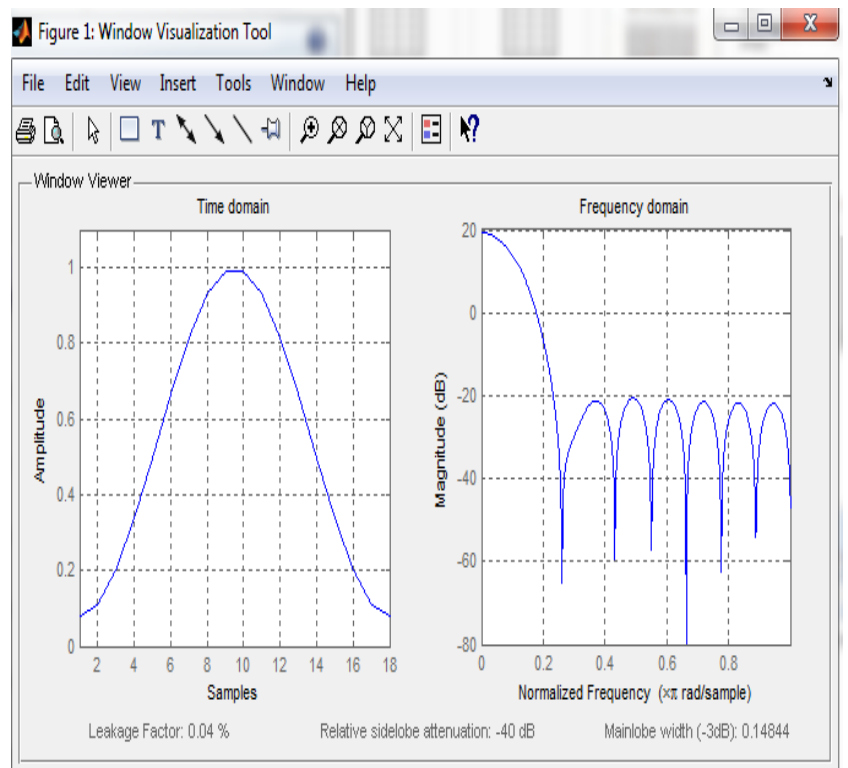

Figure no.8 feature extraction of abnormal signal

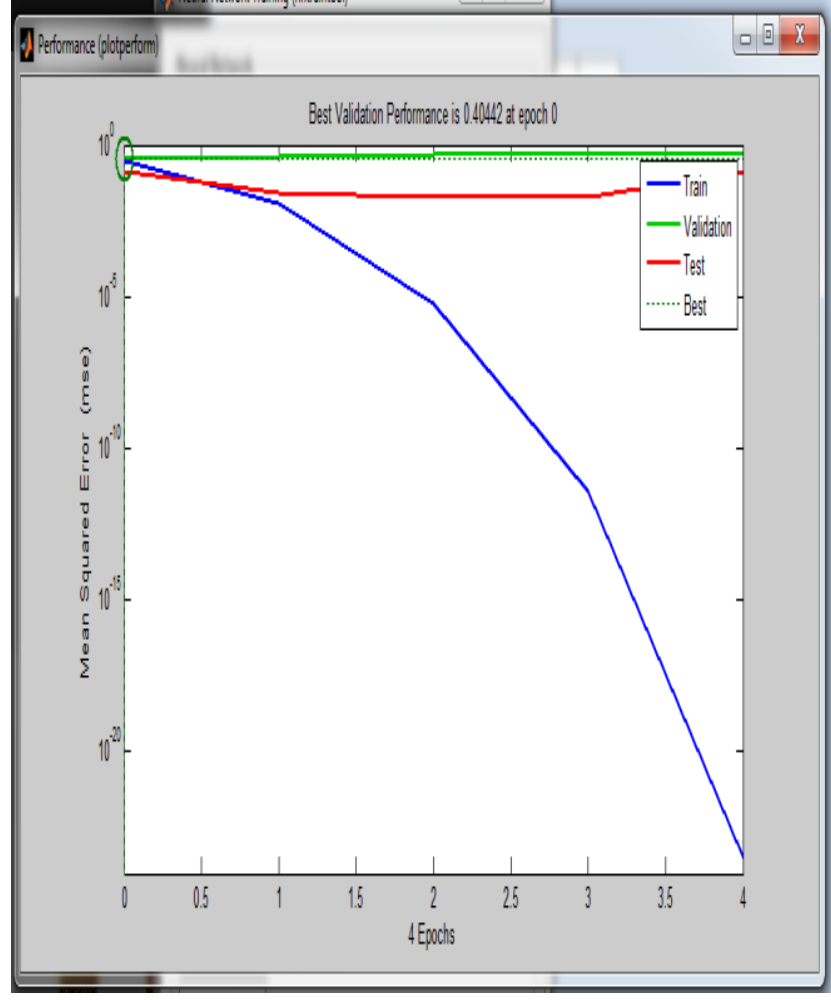

Figure 9 Performance plot for normal signal of EEG

\subsection{Test using neural network}

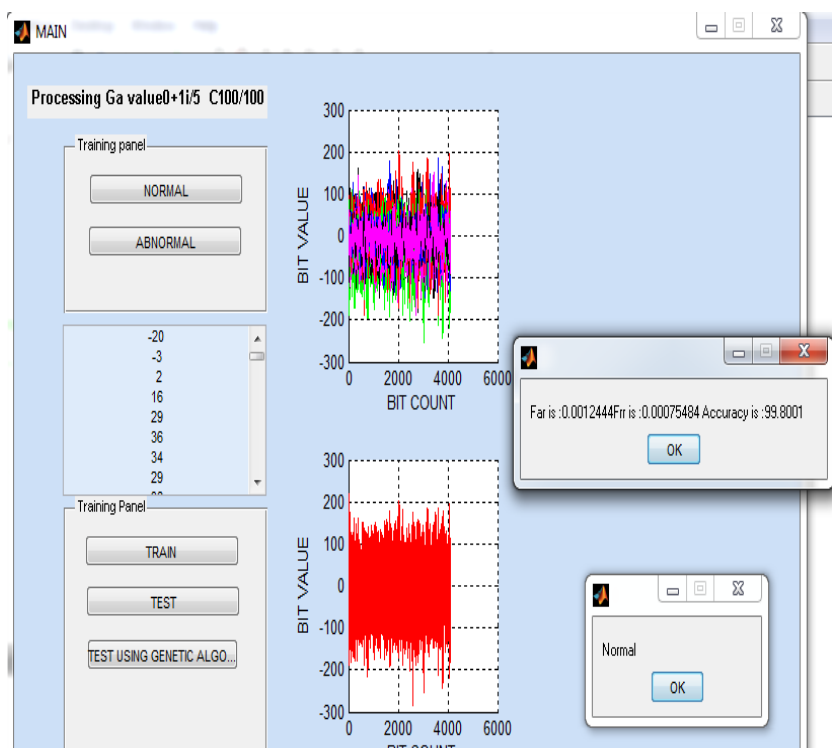

Figure 10 For normal EEG signal

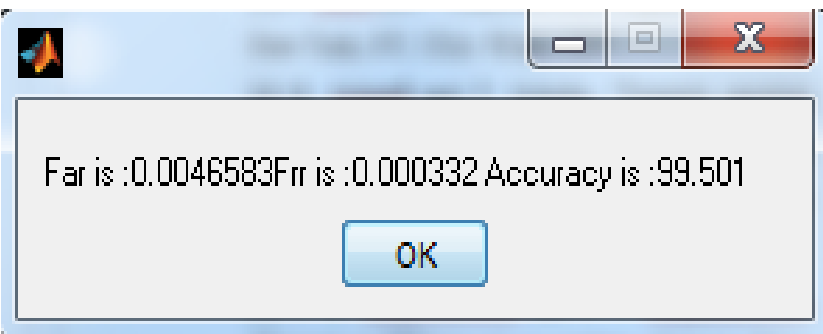

Fig.11 Evaluated Parameters by testing neural network 


\subsection{Testing using genetic algorithm}

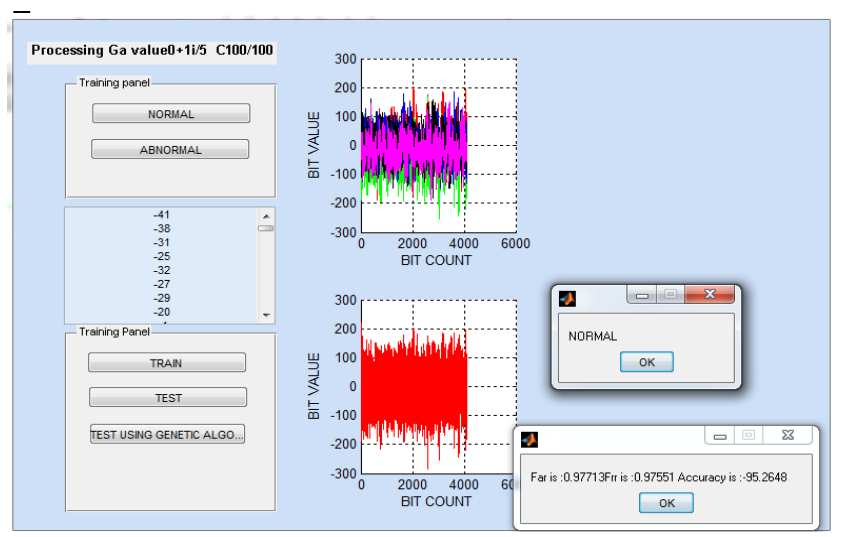

Figure 12 Test using GA for abnormal EEG signal

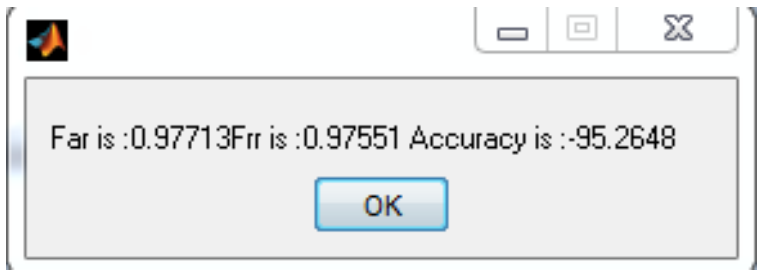

Fig.13 Evaluated Parameters by testing Genetic algorithm

Table no.2 results after testing with different algorithm

\begin{tabular}{|c|c|c|c|c|c|}
\hline s.no. & TESTING & FAR & FRR & $\begin{array}{c}\text { ACCU } \\
\text { RACY }\end{array}$ & SIGNAL \\
\hline 1 & $\begin{array}{c}\text { Using } \\
\text { neural } \\
\text { network }\end{array}$ & $\begin{array}{c}0.01031 \\
3\end{array}$ & $\begin{array}{c}0.0009 \\
4637\end{array}$ & 98.8 & NORMAL \\
\hline 2 & $\begin{array}{c}\text { Using } \\
\text { neural } \\
\text { network }\end{array}$ & $\begin{array}{c}0.00827 \\
42\end{array}$ & $\begin{array}{c}0.0005 \\
3832\end{array}$ & 99.1 & $\begin{array}{c}\text { ABNORM } \\
\text { AL }\end{array}$ \\
\hline 3 & $\begin{array}{c}\text { Using } \\
\text { genetic } \\
\text { algorithm }\end{array}$ & 0.98466 & $\begin{array}{c}0.9835 \\
7\end{array}$ & -96.8 & $\begin{array}{c}\text { NORMAL } \\
4\end{array}$ \\
$\begin{array}{c}\text { Using } \\
\text { genetic } \\
\text { algorithm }\end{array}$ & 0.85757 & $\begin{array}{c}0.8486 \\
5\end{array}$ & -70.6 & $\begin{array}{c}\text { ABNORM } \\
\text { AL }\end{array}$ \\
\hline
\end{tabular}

The proposed algorithm working has been verified using three parameters i.e. FAR (False Acceptance Ratio), FRR (False Rejection Ratio) and Accuracy. It has been concluded that $99.501 \%$ efficiency has been achieved by the neural network technique and by genetic it gives negative values.

\section{CONCLUSION AND FUTURE SCOPE}

This paper has presented the feature extraction and classification of EEG signals using machine learning algorithm. Genetic algorithm is used to select the best combination of features that minimize classification error. An important disadvantage of using genetic algorithm in practice is the long time it requires to complete calculations. Neural network as well as genetic algorithm feature extraction techniques have been used in this method too. Using these features, it has been concluded that which of the EEG data is normal or abnormal. This emphasize on the classification as well as feature extraction part so that it will be helpful in emotion recognition. Ideally, a good classifier for BCI should produce high classification accuracy with minimal computational complexity and feature extraction technique must extract features precisely. So in this work neural network and above mentioned features has been extracted to get high accuracy. From the results it has been concluded that by obtaining values for $\mathrm{FAR}=.004, \mathrm{FAR}=.0003$ and accuracy $=99.55$ shows that proposed algorithm is good working technique for this type of problem specification.

Further work suggestions include the use of the BPNN (Back Propagation Neural Network) for training purpose. As in the present work training has been done using Levenburg Neural Network. As it have wide range of operating conditions and less complexity. If the signal is non-stationary, timefrequency methods can bring up additional information by considering dynamical changes.

\section{REFERENCES}

[1] M.-K. Kim, M. Kim, E. Oh, and S.-P. Kim, "A review on the computational methods for emotional state estimation from the human EEG," Comput. Math. Methods Med., vol. 2013, pp. 1-13, Jan. 2013

[2] J. T. Cacioppo, "Feelings and emotions: Roles for electrophysiological\ markers," Biol. Psychol., vol. 67, no. $1-2$, pp. $235-43$, Oct. 2004.

[3] S. Sanei and J. Chambers, EEG Signal Processing. New York, NY, USA: Wiley, 2007.

[4] K. Schaaff and T. Schultz, "Towards emotion recognition from electroencephalographic signals," in Proc. Int. Conf. Affect. Comput. Intell. Interact., Sep. 2009, pp. 175-180.

[5] S. K. Hadjidimitriou and L. J. Hadjileontiadis, "Toward an EEG-based recognition of music liking using timefrequency analysis," IEEE Trans. Biomed. Eng., vol. 59, no. 12, pp. 3498-510, Dec. 2012.

[6] P. C. Petrantonakis and L. J. Hadjileontiadis, "Emotion recognition from EEG using higher order crossings," IEEE Trans. Inf. Technol. Biomed., vol. 14, no. 2, pp. 186-197, Mar. 2010.

[7] K. Takahashi, "Remarks on emotion recognition from multimodal bio-potential signals," in Proc. Int. Conf. Ind. Technol., 2004, pp. 1138-1143.

[8] M. Murugappan, M. Rizon, S. Yaacob, I. Zunaidi, and D. Hazry, "EEG feature extraction for classifying emotions using FCM and FKM," Int. J. Comput. Commun., vol. 1, no. 2, pp. 21-25, 2007.

[9] X. Wang, D. Nie, and B. Lu, "EEG-based emotion recognition using frequency domain features and support vector machines," in Proc. Int. Conf. Neural Inf. Process., 2011, pp. 734-743.

[10] K. Ansari-asl, G. Chanel, and T. Pun, "A channel selection method for EEG classification in emotion assessment based on synchronization likelihood," in Proc. 15th Eur. Signal Process.Conf., 2007, pp. 12411245. 
[11] R. Jenke, A. Peer, and M. Buss, "Effect-size-based electrode and feature selection for emotion recognition from EEG," in Proc. IEEE Int. Conf. Acoustics, Speech Signal Process., 2013, pp. 1217-1221.

[12] C. Frantzidis, C. Bratsas, C. Papadelis, E. Konstantinidis, C. Pappas, and P. Bamidis, "Toward emotion aware computing: An integrated approach using multichannel neurophysiological recordings and affective visual stimuli," IEEE Trans. Inf. Technol. Biomed., vol. 14, no. 3, pp. 589-597, May 2010.

[13] Y. Liu and O. Sourina, "Real-time fractal-based valence level recognition from EEG," Trans. Comput. Sci. XVIII, vol. 7848, pp. 101-120, 2013.

[14] M. Murugappan, R. Nagarajan, and S. Yaacob, "Classification of human emotion from EEG using discrete wavelet transform," J. Biomed. Sci. Eng., vol. 3, no. 4, pp. 390-396, 2010.
[15] E. Kroupi, A. Yazdani, and T. Ebrahimi, "EEG correlates of different emotional states elicited during watching music videos," in Proc. Int. Conf. Affect. Comput. Intell. Interact. 2011, pp. 457-466.

[16] R. Horlings, D. Datcu, and L. Rothkrantz, "Emotion recognition using brain activity," in Proc. Int. Conf. Comput. Syst. Technol., 2008, pp. II.1-1-6.

[17] J. Hausdorff, A. Lertratanakul, M. Cudkowicz, A. Peterson, D. Kaliton, and A. Goldberger, "Dynamic markers of altered gait rhythm in amyotrophic lateral sclerosis,” J. Appl. Physiol., vol. 88, pp. 2045-2053, 2000.

[18] R. Khosrowabadi and A. Rahman, "Classification of EEG correlates on emotion using features from Gaussian mixtures of EEG spectrogram," in Proc. 3rd Int. Conf. Inf. Commun. Technol. Moslem World, 2010, pp. E102E107. 